\title{
A mid-pregnancy risk prediction model for gestational diabetes mellitus based on the maternal status in combination with ultrasound and serological findings
}

\author{
YA-ZHONG ZHANG $^{1 *}$, LEI ZHOU ${ }^{1 *}$, LUOBING TIAN $^{1}$, XIN LI $^{2}$, \\ GUYUE ZHANG $^{1}$, JIANG-YUAN QIN ${ }^{1}$, DAN-DAN ZHANG ${ }^{1}$ and HUI FANG ${ }^{1}$ \\ Departments of ${ }^{1}$ Endocrinology and ${ }^{2}$ Imaging, Tangshan Gongren Hospital, Tangshan, Hebei 063000, P.R. China
}

Received September 4, 2019; Accepted February 28, 2020

DOI: $10.3892 /$ etm. 2020.8690

\begin{abstract}
Although previous studies have proposed predictive models of gestational diabetes mellitus (GDM) based on maternal status, they do not always provide reliable results. The present study aimed to create a novel model that included ultrasound data of maternal fat distribution and serum inflammatory factors. The clinical data of 1,158 pregnant women treated at Tangshan Gongren Hospital and eight other flagship hospitals in Tangshan, including the First Hospital of Tangshan Gongren Hospital group, Ninth Hospital of Tangshan Gongren Hospital group, Tangshan Gongren Hospital group rehabilitation hospital, Tangshan railway central hospital, Tangshan Gongren Hospital group Fengnan hospital, Tangshan Gongren Hospital group Qianan Yanshan hospital, Tangshan Gongren Hospital group Qianxi Kangli hospital and Tangshan Gongren Hospital group Jidong Sub-hospital, were analyzed following the division of subjects into GDM and non-GDM groups according to their diagnostic results at 24-28 weeks of pregnancy. Univariate analysis was performed to investigate the significance of the maternal clinical parameters for GDM diagnosis and a GDM prediction model was established using stepwise regression analysis. The predictive value of the model was evaluated using a Homer-Lemeshow goodness-of-fit test and a receiver operating characteristic curve (ROC). The model demonstrated that age, pre-pregnancy body mass index, a family history of diabetes mellitus, polycystic ovary syndrome, a history of GDM, high systolic pressures, glycosylated hemoglobin levels, triglyceride levels, total cholesterol levels, low-density
\end{abstract}

Correspondence to: Dr Hui Fang, Department of Endocrinology, Tangshan Gongren Hospital, 27 Wenhua Road, Lubei, Tangshan, Hebei 063000, P.R. China

E-mail: fanghui0504@sina.cn

*Contributed equally

Key words: gestational diabetes mellitus, risk factors, risk prediction model lipoprotein cholesterol levels, serum hypersensitive C-reactive protein, increased subcutaneous fat thickness and visceral fat thickness were all correlated with an increased GDM risk (all $\mathrm{P}<0.01)$. The area under the curve value was 0.911 (95\% CI, 0.893-0.930). Overall, the results indicated that the current model, which included ultrasound and serological data, may be a more effective predictor of GDM compared with other single predictor models. In conclusion, the present study developed a tool to determine the risk of GDM in pregnant women during the second trimester. This prediction model, based on various risk factors, demonstrated a high predictive value for the GDM occurrence in pregnant women in China and may prove useful in guiding future clinical practice.

\section{Introduction}

Gestational diabetes mellitus (GDM) is defined as impaired glucose tolerance in the second or third trimester of pregnancy, with no apparent manifestation of diabetes during pre-pregnancy (1). GDM is an increasingly common pregnancy-associated complication (2), with an incidence as high as $18-36 \%$ according to worldwide epidemiological surveys $(3,4)$. GDM is associated with various adverse maternal and fetal outcomes, including pregnancy-induced hypertension, pre-eclampsia, macrosomia, neonatal hypoglycemia and shoulder dystocia $(5,6)$. Concurrently, women with GDM have an increased risk of developing type 2 diabetes mellitus (T2DM) post-pregnancy (7).

Previous studies have determined various risk factors associated with GDM including maternal characteristics, age, family history of diabetes and polycystic ovary syndrome (PCOS) (8-10). Currently, clinical management guidelines for obstetrician-gynecologists produced by The American College of Obstetricians and Gynecologists recommend early pregnancy glucose screening for women at a high risk of increased blood glucose levels (1), as well as for those with risk factors such as hypertension, glycated hemoglobin levels (HbA1c) $\geq 5.7 \%$, impaired glucose tolerance or impaired fasting glucose, a high-density lipoprotein cholesterol level $\leq 35 \mathrm{mg} / \mathrm{dl}$ and triglyceride (TG) levels $\geq 250 \mathrm{mg} / \mathrm{dl}$ (7). Additionally, the $2 \mathrm{~h} 75 \mathrm{~g}$ oral glucose tolerance test remains the gold standard for the clinical diagnosis of GDM at 24 weeks of gestation (1). 
A recent study has proposed a formula to predict the probability of GDM between 8 and 20 weeks of gestation based on maternal age, pre-pregnancy body mass index (BMI), fasting plasma glucose and TG levels (11). Additionally, numerous GDM predictive tools incorporate information on maternal and biochemical factors (12). However, these traditional screening methods are based on the mother's medical history, which can demonstrate a high false-positive rating for predicting the disease (13).

To the best of our knowledge, there are currently no established criteria or models for predicting GDM that combine sociodemographic characteristics, serological indicators and ultrasound examinations. The early identification of risk factors in pregnant women may help to predict the occurrence of GDM prior to clinical diagnosis. Thus, there is an urgent requirement to determine effective ways to predict the future development of GDM. The present study aimed to develop a useful predictive tool for the early identification of high-risk pregnant women, which could provide a scientific basis for the early identification of high-risk patients with GDM.

\section{Materials and methods}

Patient studies. The current study was approved by the Institutional Review Board of Tangshan Gongren Hospital and written informed consent was obtained from all patients. All procedures and methods were performed in accordance with the approved guidelines.

From January 2016 to May 2018, a total of 4,421 pregnant women (mean age 27.5 \pm 6.2 years, range 20-45) who received routine pre-natal examinations at Tangshan Gongren Hospital and eight other flagship hospitals (including First Hospital of Tangshan Gongren Hospital group, Ninth Hospital of Tangshan Gongren Hospital group, Tangshan Gongren Hospital group rehabilitation hospital, Tangshan railway central hospital, Tangshan Gongren Hospital group Fengnan hospital, Tangshan Gongren Hospital group Qianan Yanshan hospital, Tangshan Gongren Hospital group Qianxi Kangli hospital and Tangshan Gongren Hospital group Jidong Sub-hospital) were observed.

The inclusion criteria included: i) Pregnancy at 12-16 weeks gestation; and ii) GDM diagnosis according to the 2017 GDM diagnostic criteria by the American Diabetes Association (1). The exclusion criteria were: i) Pregnancy with heart or cerebrovascular disease or vital organ dysfunction; ii) prior antidiabetic medication; and/or iii) pre-existing diabetes diagnosis [fasting blood glucose (BGL) levels $\geq 7.0 \mathrm{mmol} / 1$, HbAlc levels $\geq 6.5 \%$ and/or random blood glucose levels $\geq 11.1 \mathrm{mmol} / \mathrm{l}]$. During testing, 737 women were not eligible when assessed. 2346 women declined to participate in the trial.103 women quit the trial due to termination or miscarriages. For a variety of reasons, 99 women didn't undergo a $75 \mathrm{~g}$ oral glucose tolerance test (OGTT) at 24-28 weeks of gestation.

After further screening, selected pregnant women underwent an OGTT between 24-28 weeks of gestation. Blood samples were collected from patients for glucose and insulin measurement at $0,0.5,1$ and $2 \mathrm{~h}$ after administration of OGTT to diagnose GDM. Of these patients, 1,136 were enrolled in the present study and included 406 women (35.75\%) diagnosed with GDM and 730 women (64.25\%) that were negative for
GDM (non-GDM; Fig. 1). Medical records of these enrolled patients at 12-16 weeks of gestation were retrospectively evaluated.

Data collections. Questionnaires were used to collect baseline clinical data from pregnant women before 12 weeks of pregnancy, including age, education, occupation, BMI, family history, parity, a history of PCOS or of previous GDM, systolic blood pressure (SBP), diastolic blood pressure and BGL levels. Right brachial artery blood pressure was measured using a corrected mercury sphygmomanometer between 7:00 and 9:00 a.m., with individuals required to sit and rest for $15 \mathrm{~min}$ prior to measurement. Occupations were divided into light work (clerks and civil servants), medium work (teachers, students and self-employed) or heavy work (farmers, medical staff and military personnel) according to the China Physical Labor Intensity Grading Standard (14).

Laboratory tests. HbA1c were measured at 12-16 weeks of gestation using high-performance liquid chromatography (HPLC) with a cation exchange chromatography column (Bio-Rex70, 200x6 mm, Bio-Rad,Hercules, CA, USA). $15 \mu$ l of sample volume was injected into the HPLC system. The HPLC system (, Waters, Milford, MA, USA) was operated at a column temperature of $40^{\circ} \mathrm{C}$ and a flow rate of $1 \mathrm{ml} / \mathrm{min}$. Mobile phase composition was as follows: mobile phase A, PBS (pH6.6,40 mmol/l); mobile phase $\mathrm{B}, \mathrm{NaCl}$ (pH 6.4, $300 \mathrm{mmol} / \mathrm{l})$. TG, total cholesterol (TC) and low-density lipoprotein cholesterol (LDL-c) levels and liver function tests, including measuring alanine transaminase (ALT), aspartate transaminase (AST) and transglutaminase (GGT) plasma levels, were performed using an automatic biochemistry analyzer (model AU600, Olympus Corporation; 7150, Hitachi, Ltd.) in the nine study center laboratories (including central laboratory of Tangshan Gongren Hospital, laboratory of First Hospital of Tangshan Gongren Hospital group, laboratory of Ninth Hospital of Tangshan Gongren Hospital group, laboratory of Tangshan Gongren Hospital group rehabilitation hospital, laboratory of Tangshan railway central hospital, laboratory of Tangshan Gongren Hospital group Fengnan hospital, laboratory of Tangshan Gongren Hospital group Qianan Yanshan hospital, laboratory of Tangshan Gongren Hospital group Qianxi Kangli hospital and laboratory of Tangshan Gongren Hospital group Jidong Sub-hospital). Serum hypersensitive C-reactive protein (hs-CRP) was measured using immunoturbidimetry (Cias Latex CRP-H kit; Kanto Chemical Co., Inc.). Serum visfatin and adiponectin levels were analyzed using ELISA kits (Phoenix Pharmaceuticals, Inc.; cat. no. EK-003-80 and EK-ADI-01, respectively).

Ultrasonography. A high-resolution Philips IU22 ultrasound system (Philips Healthcare) equipped with a $3.5-10.0 \mathrm{MHz}$ wide-band convex sensor was used for ultrasonic measurements. To avoid any influence caused by abdominal wall tension due to inhalation, abdominal ultrasound examinations were performed in the supine position during deep inhalation. Subcutaneous fat thickness (SFT) was determined as the thickness of the fat between the liver surface and the abdominal white line and visceral fat thickness (VFT) was defined as the thickness of the fat between the skin-fat interface and the white line (15). 


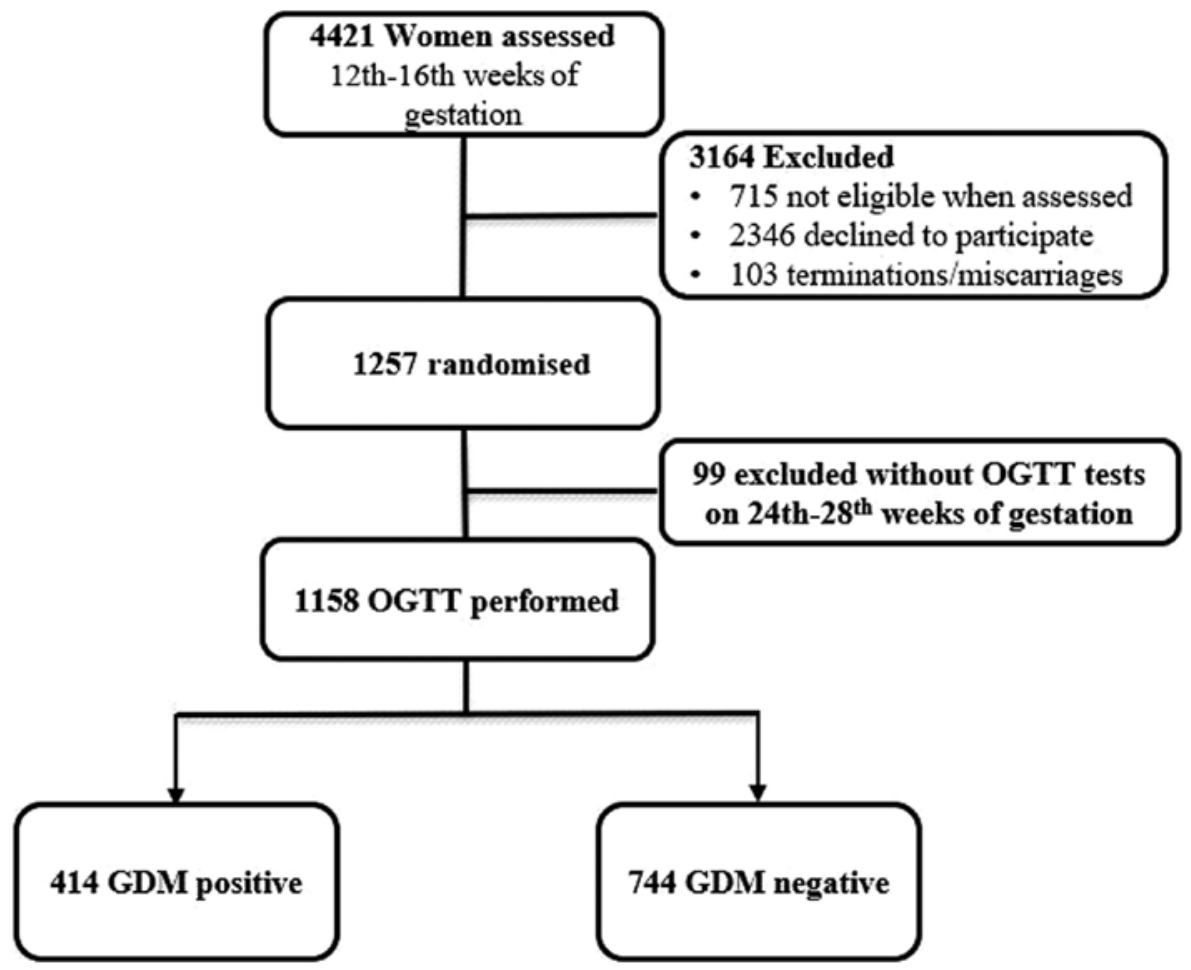

Figure 1. Flow chart illustrating the recruitment of patients with GDM and controls. GDM, gestational diabetes; OGTT, $75 \mathrm{~g}$ oral glucose tolerance test.

Statistical analysis. Data were analyzed using SPSS software (version 13.0; SPSS, Inc.) and expressed as the mean \pm standard deviation or the median and the interquartile range. Statistical differences between non-GDM and GDM groups were determined using Student's $t$-test or Wilcoxon test. Count data are expressed as numbers and percentages and were evaluated using Fisher's exact probability method. $\mathrm{P}<0.05$ was considered to indicate a statistically significant difference.

To assess the predictive variables of GDM, a logistic regression model was established following adjustments for age, pre-pregnancy BMI, a family history of diabetes, PCOS, a history of GDM, SBP, and HbA1c, TG, TC, LDL-c, ALT, AST, GGT, visfatin, hs-CRP, VFT and SFT levels. Logistic regression was used to determine GDM risk predictors. According to previous reports (16), the present study used the stepwise forward selection method as the variable selection procedure. Based on the results of logistic regression, the predictability of the model was evaluated to give $95 \%$ confidence intervals (CIs) and P-values. The logistic regression model was evaluated using the area under the curve (AUC) of the receiver operating characteristic curve and the Homer-Lemeshow goodness-of-fit test was used to verify the efficiency of the prediction model.

\section{Results}

Clinical data and biochemical results. The sociodemographic and clinical characteristics of patients in the non-GDM and GDM groups are summarized in Table I. The patients ranged in age from 20-45 years (GDM average; 28.69 \pm 4.73 ; non-GDM average; $26.40 \pm 3.64$ ) and the average pre-pregnancy BMI was $24.82 \pm 2.28$ and $23.08 \pm 1.66 \mathrm{~kg} / \mathrm{m}^{2}$ for the GDM and non-GDM groups, respectively. In addition, there was no significant difference in liver function between the two groups.
Compared with women in the non-GDM group, women in the GDM group were older, demonstrated higher BMIs. were more prone to PCOS, exhibited a family history of diabetes and were more likely to present with a previous history of GDM. Additionally, the GDM group demonstrated higher SBP, HbAlc, TGs, TC, LDL-c, visfatin, hs-CRP, VFT and SFT (all $\mathrm{P}<0.01)$ compared with the non-GDM group.

Results of logistic regression analysis. Considering the strong association of numerous risk factors and GDM, the forward stepwise variable-selection approach was used to analyze independent predictors. The results revealed that age, pre-pregnancy BMI, a family history of diabetes, a history of GDM, PCOS, SBP and levels of TG, HbA1c, SBP, TC, LDL-c, visfatin, hs-CRP, VFT and SFT should be included in the model as these were all observed to be independent risk factors of GDM (all P<0.01; Table II).

Probability of basic and extended prediction models. Model A retained 10 variables including age, pre-pregnancy BMI, a family history of diabetes, GDM history, PCOS history, and levels of HbA1C, SBP, TG, TC and LDL-c. Model B included the above variables in addition to VFT and SFT and model C contained all variables in model B plus visfatin and hs-CRP levels. The AUC of model A was 0.846, whereas the addition of VFT and SFT significantly increased the AUC of model $\mathrm{B}$ to 0.885 (Fig. 2). However, the addition of visfatin and hs-CRP in model C (AUC, 0.911; 95\% CI, 0.893-0.930) only slightly improved the performance of model B (AUC, 0.885; 95\% CI, 0.864-0.906). All three models were well-calibrated (Table III). Regression model C was the best performing regression model with highly sensitive and strong predictive power. 
Table I. Sociodemographic and clinical characteristics of patients in the non-GDM and GDM groups (mean \pm standard deviation).

\begin{tabular}{|c|c|c|c|c|}
\hline Variable & Non-GDM group $(n=730)$ & GDM group $(n=406)$ & $t / \chi^{2}$ & P-value \\
\hline Age (years) & $26.40 \pm 3.64$ & $28.69 \pm 4.73$ & -8.45 & $<0.01$ \\
\hline BMI pregnancy $\left(\mathrm{kg} / \mathrm{m}^{2}\right)$ & $23.08 \pm 1.66$ & $24.82 \pm 2.28$ & -13.52 & $<0.01$ \\
\hline Family history of diabetes mellitus (n, \%) & $26(3.6)$ & $80(19.7)$ & 80.36 & $<0.01$ \\
\hline History of GDM $(\mathrm{n}, \%)$ & $9(1.2)$ & $25(6.2)$ & 23.73 & $<0.01$ \\
\hline Education $(\mathrm{n}, \%)$ & & & 3.54 & 0.17 \\
\hline High school or below & $369(50.5)$ & $203(50.0)$ & & \\
\hline Diploma or undergraduate & $301(41.2)$ & $181(44.6)$ & & \\
\hline Postgraduate and above & $60(8.2)$ & $22(5.4)$ & & \\
\hline Occupation (n, \%) & & & 4.42 & 0.11 \\
\hline Light labor & $371(50.8)$ & $204(50.2)$ & & \\
\hline Medium labor & $292(40.0)$ & $178(43.8)$ & & \\
\hline Heavy labor & $67(9.2)$ & $24(5.9)$ & & \\
\hline Parity (n, \%) & & & 0.27 & 0.60 \\
\hline Primipara & $373(51.1)$ & $214(52.7)$ & & \\
\hline Multipara & $357(48.9)$ & $192(47.3)$ & & \\
\hline $\operatorname{PCOS}(\mathrm{n}, \%)$ & $140(19.2)$ & $142(35.0)$ & 34.89 & $<0.01$ \\
\hline $\operatorname{HbA1c}(\%)$ & $5.66 \pm 0.35$ & $6.11 \pm 1.43$ & -6.28 & $<0.01$ \\
\hline $\begin{array}{l}\text { Fasting blood sugar of pregnant women } \\
\text { prior to } 12 \text { weeks of gestation }(\mathrm{mmol} / \mathrm{l})\end{array}$ & $5.08 \pm 1.21$ & $5.11 \pm 1.39$ & -0.37 & 0.71 \\
\hline $\mathrm{SBP}(\mathrm{mmHg})$ & $117.17 \pm 11.04$ & $120.57 \pm 8.22$ & -5.90 & $<0.01$ \\
\hline $\mathrm{DBP}(\mathrm{mmHg})$ & $80.07 \pm 10.14$ & $79.36 \pm 8.89$ & 1.24 & 0.22 \\
\hline Triglycerides (mmol/l) & $1.81 \pm 0.70$ & $1.94 \pm 0.84$ & -2.75 & $<0.01$ \\
\hline Total cholesterol (mmol/l) & $5.20 \pm 0.72$ & $6.29 \pm 1.59$ & -13.23 & $<0.01$ \\
\hline LDL cholesterol (mmol/l) & $1.94 \pm 0.99$ & $2.67 \pm 1.11$ & -11.04 & $<0.01$ \\
\hline $\operatorname{ALT}(\mathrm{mmol} / \mathrm{l})$ & $17.60 \pm 3.14$ & $17.43 \pm 3.34$ & 0.83 & 0.41 \\
\hline AST (mmol/l) & $17.10 \pm 3.18$ & $17.28 \pm 2.82$ & -0.99 & 0.32 \\
\hline GGT (mmol/l) & $18.87 \pm 2.10$ & $19.24 \pm 4.38$ & -1.63 & 0.10 \\
\hline hs-CRP (mg/l) & $1.69 \pm 0.17$ & $2.16 \pm 0.60$ & -15.63 & $<0.01$ \\
\hline Visfatin (ng/ml) & $8.98 \pm 1.09$ & $10.28 \pm 1.43$ & -16.01 & $<0.01$ \\
\hline Adiponectin $(\mu \mathrm{g} / \mathrm{ml})$ & $2,234.04 \pm 942.99$ & $2,257.65 \pm 921.80$ & -0.41 & 0.69 \\
\hline VFT (mm) & $7.64 \pm 0.61$ & $8.00 \pm 1.17$ & -5.69 & $<0.01$ \\
\hline SFT (mm) & $10.38 \pm 1.22$ & $11.38 \pm 2.49$ & -7.58 & $<0.01$ \\
\hline
\end{tabular}

GDM, gestational diabetes mellitus; BMI, body mass index; PCOS, polycystic ovary syndrome; HbA1c, glycated hemoglobin; SBP, systolic blood pressure; DBP, diastolic blood pressure; LDL, low-density lipoprotein; ALT, alanine transaminase; AST, aspartate transaminase; GGT, transglutaminase; hs-CRP, serum hypersensitive C-reactive protein; VFT, visceral fat thickness; SFT, subcutaneous fat thickness.

\section{Discussion}

In recent years, advanced etiological studies have revealed GDM to be a multifactorial pregnancy disease which can be induced by genetic factors, insulin resistance (IR), inflammation and adipokine involvement (17). In a case-controlled study reporting GDM incidence, the prevalence of GDM in pregnant Chinese women was between 6.8-10.4\%, with increased prevalence in the Beijing area at $19.7 \%$ (18). Furthermore, it has been reported that in woman $>25$ years old, the incidence of GDM increases with age (19). This is due to the placenta secreting insulin-like substances that induce IR, which occurs alongside the age-associated decreased function of islet $\beta$-cells (20).
Numerous studies have reported that increases in pre-pregnancy BMI are independent risk factors for GDM $(12,21)$. During pregnancy, the secretion of insulin-antagonistic hormones rapidly increases in obese women, which in turn increases the risk of GDM (22). The present study reported that women in the GDM group were older, had higher BMIs and higher Hbalc levels compared with the non-GDM group. Multivariate logistic regression analysis demonstrated that these variables were significantly associated with an increased risk of GDM.

Previous research has shown that pregnant women with a history of diabetes hav a 3-fold higher risk of developing GDM and this association is not affected by other confounding factors $(20,23)$. In addition, the fetuses of women with GDM may be subjected to a hyperglycemic environment during 
Table II. Logistic regression analysis for the clinical risk prediction model (mean \pm standard deviation).

\begin{tabular}{lccccc}
\hline Variable & $\beta$ & S.E & Wald & OR (95\% CI) & P-value \\
\hline Age (years) & 0.154 & 0.027 & 33.445 & $1.166(1.107-1.228)$ & $<0.01$ \\
BMI pre-pregnancy & 0.580 & 0.063 & 84.959 & $1.787(1.579-2.0212)$ & $<0.01$ \\
Family history of diabetes mellitus & -1.071 & 0.424 & 6.377 & $0.343(0.149-0.787)$ & 0.01 \\
History of GDM & -1.791 & 0.604 & 8.799 & $0.167(0.051-0.545)$ & $<0.01$ \\
PCOS & -0.839 & 0.288 & 8.476 & $0.432(0.246-0.7600)$ & $<0.01$ \\
HbA1c (\%) & 0.833 & 0.207 & 16.134 & $2.299(1.532-3.452)$ & $<0.01$ \\
SBP (mmHg) & 0.030 & 0.011 & 7.499 & $1.030(1.009-1.053)$ & $<0.01$ \\
Triglycerides (mmol/l) & 0.432 & 0.140 & 9.515 & $1.541(1.171-2.028)$ & $<0.01$ \\
Total cholesterol (mmol/l) & 1.046 & 0.163 & 41.017 & $2.846(2.066-3.919)$ & $<0.01$ \\
LDL cholesterol (mmol/l) & 0.579 & 0.103 & 31.887 & $1.784(1.459-2.182)$ & $<0.01$ \\
hs-CRP (mg/l) & 1.427 & 0.296 & 23.212 & $4.165(2.331-7.441)$ & $<0.01$ \\
Visfatin (ng/ml) & 0.891 & 0.098 & 83.080 & $2.438(2.013-2.952)$ & $<0.01$ \\
VFT (mm) & 0.760 & 0.133 & 32.574 & $2.139(1.647-2.776)$ & $<0.01$ \\
SFT (mm) & 0.495 & 0.066 & 56.629 & $1.641(1.443-1.867)$ & $<0.01$ \\
\hline
\end{tabular}

GDM, gestational diabetes mellitus; S.E, standard error; OR, odds ratio; CI, confidence interval; BMI, body mass index; PCOS, polycystic ovary syndrome; HbA1c, glycated hemoglobin; SBP, systolic blood pressure; LDL, low-density lipoprotein; hs-CRP, serum hypersensitive C-reactive protein; VFT, visceral fat thickness; SFT, subcutaneous fat thickness.

Table III. Performance of different models predicting gestational diabetes mellitus.

\begin{tabular}{lcccc}
\hline Model & Sensitivity (\%) & Specificity (\%) & Positive predictive value (\%) & Negative predictive value (\%) \\
\hline A & 70.0 & 91.8 & 82.56 & 84.60 \\
B & 76.6 & 92.1 & 84.28 & 87.61 \\
C & 83.0 & 94.7 & 89.63 & 90.92 \\
\hline
\end{tabular}

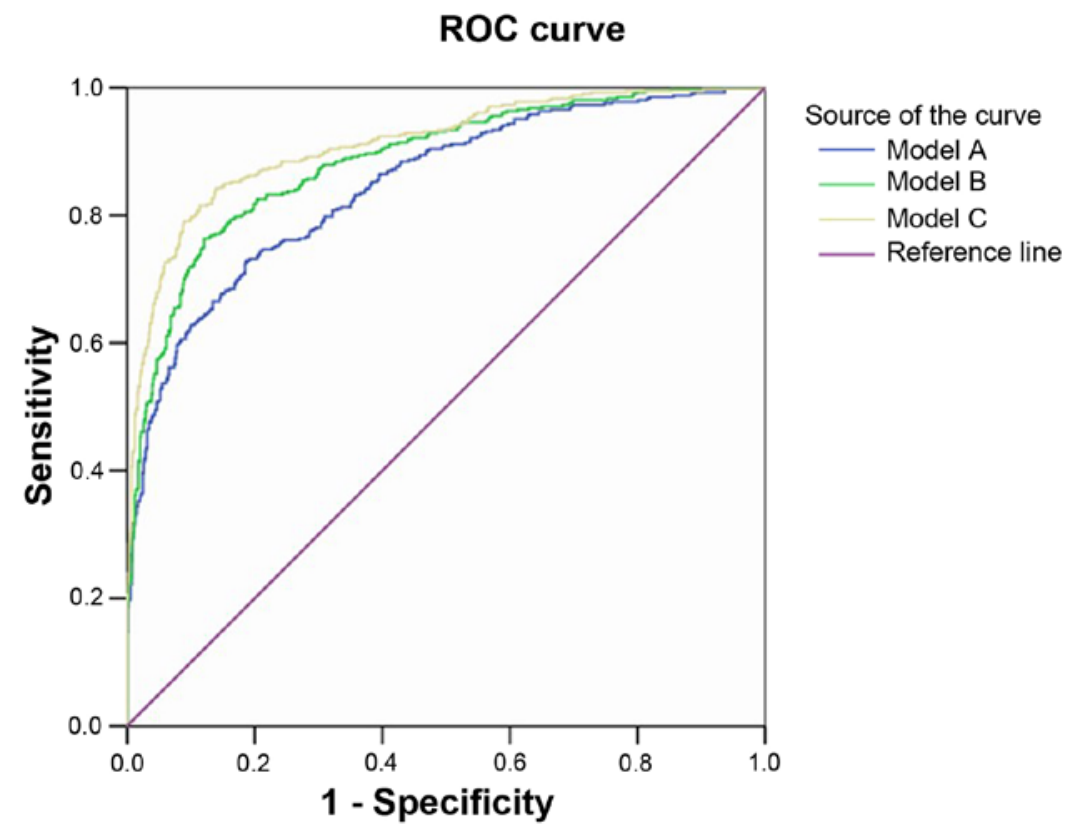

Figure 2. ROC curves of three different GDM prediction models. Model A included the following variables: Age, pre-pregnancy BMI, a family history of diabetes, GDM history, PCOS history, and levels of HbA1C, SBP, TG, TC and LDL-c. Model B included the following variables: Model A plus VFT and SFT. Model C included the following variables: Model B plus visfatin and hs-CRP. ROC, receiver operating characteristic; GDM, gestational diabetes mellitus; BMI, body mass index; PCOS, polycystic ovary syndrome; HBA1C, glycated hemoglobin; SBP, systolic blood pressure; TG, triglyceride; TC, total cholesterol; LDL-c, low-density lipoprotein cholesterol; VFT, visceral fat thickness; SFT, subcutaneous fat thickness; hs-CRP, serum hypersensitive C-reactive protein. 
pregnancy that stimulates fetal islet $\beta$ cell proliferation and abnormal proliferation of adipocytes (24). These fetuses tend to develop obesity in adulthood and are prone to abnormal glucose metabolism during their own pregnancies. The results of the present study reported that women with GDM were more likely to have a family history of T2DM and a previous history of GDM, and were therefore included in the final regression model. A previous study has suggested that family history of T2DM influences GMD occurrence partially due to overlapping genetic bases between the diseases (25).

GDM and PCOS are common endocrine diseases in women of childbearing age, which are associated with central obesity and IR $(26,27)$. It has been suggested that PCOS may be an early-stage manifestation of various IR-associated diseases, including GDM, with the results of the present study confirming that PCOS is independently associated with GDM (28). In addition, a second study demonstrated that PCOS has a long-term effect on glucose metabolism (29). Women with PCOS and GDM were more likely to have impaired fasting blood glucose or impaired glucose tolerance at 18 months post-delivery compared with women that only exhibited GDM (29). Furthermore, hyperandrogenism with accompanying anovulation is one of the main features of PCOS and a previous study has demonstrated that patients with PCOS had lower levels of free androgen and sex hormone-binding globulin (SHBG) compared with patients without GDM (30). A previous study also demonstrated that obese women with PCOS and low SHBG levels were more likely to develop GDM compared with those with high SHBG levels (31).

During early pregnancy, estrogen and progesterone stimulate maternal $\beta$ cell proliferation and insulin secretion, which promotes maternal fat and hepatic glycogen storage to support fetal growth $(32,33)$. In the first trimester of pregnancy, insulin sensitivity may increase. However, as the pregnancy progresses to mid-pregnancy, systemic insulin sensitivity declines gradually (33). Previous studies have reported that IR is more prevalent in patients with GDM, leading to an increase in maternal blood glucose $(17,18)$. Additionally, there is evidence to suggest that IR is present in women with gestational hypertension (34) and increased blood pressure in pregnancy has been associated with the gradual impairment of glucose tolerance (35). These studies reinforce the results of the present study.

Patients with GDM have higher levels of TGs and lower insulin sensitivity (36). In addition, mRNA levels of genes involved in lipid and fatty acid metabolism are often altered in women with GDM (37). Changes in maternal lipid metabolism during pregnancy are characterized by a modest increase in lipids during early pregnancy and a significant increase in lipids during late pregnancy, particularly TGs and cholesterol (38). Lipid levels are closely associated with the dysfunction of visceral abdominal adipose tissue (39). A previous study demonstrated that central fat deposition is more closely associated with perinatal diseases (such as GDM) compared with peripheral fat accumulation (40). Ultrasounds are used to measure visceral and subcutaneous fat thickness during pregnancy due to their good practicability, objectivity and reproducibility, with VFT and SFT generally considered as appropriate to measure the distribution of visceral and subcutaneous fat. The present study observed that pregnant women with GDM had more VFT and SFT and it has been demonstrated that increased subcutaneous and visceral fat may lead to increased IR in muscle and adipose tissue (41).

Hs-CRP is an acute-phase protein associated with an increased risk of GDM (42). Given the positive association between hs-CRP and the risk of GDM development, the present study also observed that hs-CRP levels may predict GDM risk. In addition, the current study demonstrated that women with GDM exhibited higher concentrations of visfatin compared with healthy pregnant controls, which is consistent with a previous study (43). The study also reported the association between serum visfatin, fasting blood glucose, insulin and post-load insulin levels. Thus, altogether the accumulating evidence suggests that dyslipidemia and inflammation may serve an essential role in the progression of GDM.

However, as an important index of visceral fat metabolism (particularly liver fat metabolism), there was no significant difference in liver function between the two groups. This may be due to the limited sample quantity the current study. In future research, pregnant rats fed with a high fat diet could be used to study the association between liver function and adipose tissue.

In conclusion, age, pre-pregnancy BMI, a family history of diabetes, PCOS and levels of TGs, HbA1c, TC, LDL-c, visfatin, hs-CRP, VFT and SFT were all demonstrated to be independent risk factors of GDM. By analyzing maternal characteristics, sociodemographic characteristics, serological indicators and ultrasound examinations, the results of the present study suggested that this combined method adequately estimated the risk factors for GDM in the second trimester. Following the successful predictive ability of the model, it is hypothesized that the prediction model developed in the present study may represent an improved model capable of predicting GDM in women in the Tangshan area. In addition, it is noteworthy that only Tangshan Gongren Hospital and the other eight flagship hospitals in Tangshan have been included in the present study to develop the GDM risk prediction model. The results may not therefore be representative of the total population. In future studies, large-scale multi-center clinical trials are required before generalizing the results to the population of China.

\section{Acknowledgements}

Not Applicable.

\section{Funding}

The present study was supported by Tangshan Science and Technology Project (grant no. 17130224a).

\section{Availability of data and materials}

The datasets used and/or analyzed during the current study are available from the corresponding author on reasonable request.

\section{Authors' contributions}

YZ, LZ and HF conceived and designed the study. LT, XL, GZ, JQ and DZ performed data collection and ultrasonic examina- 
tion. LT and HF wrote the manuscript. DZ and HF reviewed and edited the manuscript. All authors read and approved the manuscript.

\section{Ethics approval and consent to participate}

All research was conducted with the approval of the Ethics Committee of Tangshan Gongren Hospital (clearance number GRYY-LL-2016-03). All subjects signed written informed consent.

\section{Patient consent for publication}

Not applicable.

\section{Competing interests}

The authors declare that they have no competing interests.

\section{References}

1. Committee on Practice Bulletins-Obstetrics: Practice bulletin no. 137: Gestational diabetes mellitus. Obstet Gynecol 122 406-416, 2013

2. Marathe PH Gao HX and Close KL: American diabetes association standards of medical care in diabetes 2017. J Diabetes 9: 320-324, 2017

3. Duran A, Sáenz S, Torrejón MJ, Bordiú E, Del Valle L, Galindo M, Perez N, Herraiz MA, Izquierdo N, Rubio MA, et al Introduction of IADPSG criteria for the screening and diagnosis of gestational diabetes mellitus results in improved pregnancy outcomes at a lower cost in a large cohort of pregnant women: The St. carlos gestational diabetes study. Diabetes Care 37: 2442-2450, 2014.

4. Boyadzhieva MV, Atanasova I, Zacharieva S, Tankova T and Dimitrova V: Comparative analysis of current diagnostic criteria for gestational diabetes mellitus. Obstet Med 5: 71-77, 2012.

5. Xu X, Liu Y, Liu D, Li X, Rao Y, Sharma M and Zhao Y: Prevalence and determinants of gestational diabetes mellitus: A cross-sectional study in china. Int J Environ Res Public Health 14: pii: E1532, 2017.

6. Hartling L, Dryden DM, Guthrie A, Muise M, Vandermeer B and Donovan L: Benefits and harms of treating gestational diabetes mellitus: A systematic review and meta-analysis for the US preventive services task force and the national institutes of health office of medical applications of research. Ann Intern Med 159: 123-129, 2013.

7. Shah BR, Retnakaran R and Booth GL: Increased risk of cardiovascular disease in young women following gestational diabetes mellitus. Diabetes Care 31: 1668-1669, 2008.

8. Lin PC, Hung CH, Chan TF, Lin KC, Hsu YY and Ya-Ling Tzeng: The risk factors for gestational diabetes mellitus: A retrospective study. Midwifery 42: 16-20, 2016.

9. Larrabure-Torrealva GT, Martinez S, Luque-Fernandez MA, Sanchez SE, Mascaro PA, Ingar H, Castillo W, Zumaeta R, Grande M, Motta V, et al: Prevalence and risk factors of gestational diabetes mellitus: Findings from a universal screening feasibility program in Lima, Peru. BMC Pregnancy Childbirth 18: 303, 2018.

10. Natamba BK, Namara AA and Nyirenda MJ: Burden, risk factors and maternal and offspring outcomes of gestational diabetes mellitus (GDM) in sub-Saharan Africa (SSA): A systematic review and meta-analysis. BMC Pregnancy Childbirth 19: 450, 2019.

11. Zheng T, Ye W, Wang X, Li X, Zhang J, Little J, Zhou L and Zhang L: A simple model to predict risk of gestational diabetes mellitus from 8 to 20 weeks of gestation in chinese women. BMC Pregnancy Childbirth 19: 252, 2019.

12. Sweeting AN, Wong J, Appelblom H, Ross GP, Kouru H, Williams PF, Sairanen M and Hyett JA: A novel early pregnancy risk prediction model for gestational diabetes mellitus. Fetal Diagn Ther 45: 76-84, 2019.
13. Waugh N, Scotland G, McNamee P, Gillett M, Brennan A, Goyder E, Williams R and John A: Screening for type 2 diabetes: Literature review and economic modelling. Health Technol Assess 11: iii-iv, ix-xi, 1-125, 2007.

14. State Bureau of Technical Supervision: Classification on intensity of physical work (GB 3869-1997). China Standard Press, Beijing, 1997.

15. Kaess BM, Pedley A, Massaro JM, Murabito J, Hoffmann U and Fox CS: The ratio of visceral to subcutaneous fat, a metric of body fat distribution, is a unique correlate of cardiometabolic risk. Diabetologia 55: 2622-2630, 2012.

16. Dowdy S, Weardon S and Chilko D: Multiple Regression and Correlation. In: Statistics for Research. 3rd edition. Wiley \& Sons, Inc., Hoboken, NJ, 2005.

17. Carroll X, Liang X, Zhang W, Zhang W, Liu G, Turner N and Leeper-Woodford S: Socioeconomic, environmental and lifestyle factors associated with gestational diabetes mellitus: A matched case-control study in Beijing, China. Sci Rep 8: 8103, 2018.

18. McCabe CF and Perng W: Metabolomics of diabetes in pregnancy. Curr Diab Rep 17: 57, 2017.

19. Sajani TT, Rahman MT and Karim MR: Maternal and fetal outcome of mothers with gestational diabetes mellitus attending BIRDEM hospital. Mymensingh Med J 23: 290-298, 2014.

20. Szoke E, Shrayyef MZ, Messing S, Woerle HJ, van Haeften TW, Meyer C, Mitrakou A, Pimenta W and Gerich JE: Effect of aging on glucose homeostasis: Accelerated deterioration of beta-cell function in individuals with impaired glucose tolerance. Diabetes Care 31: 539-543, 2008.

21. Lauenborg J, Hansen T, Jensen DM, Vestergaard H, Mølsted-Pedersen L, Hornnes P, Locht H, Pedersen $\mathrm{O}$ and Damm P: Increasing incidence of diabetes after gestational diabetes: A long-term follow-up in a Danish population. Diabetes Care 27: 1194-1199, 2004.

22. Adane AA, Tooth LR and Mishra GD: Pre-pregnancy weight change and incidence of gestational diabetes mellitus: A finding from a prospective cohort study. Diabetes Res Clin Pract 124: 72-80, 2017.

23. Zhu WW, Yang HX, Wang C, Su RN, Feng H and Kapur A: High prevalence of gestational diabetes mellitus in Beijing: Effect of maternal birth weight and other risk factors. Chin Med J (Engl) 130: 1019-1025, 2017.

24. Kubo A, Ferrara A, Windham GC, Greenspan LC, Deardorff J, Hiatt RA, Quesenberry CP Jr, Laurent C, Mirabedi AS and Kushi LH: Maternal hyperglycemia during pregnancy predicts adiposity of the offspring. Diabetes Care 37: 2996-3002, 2014.

25. Zhang C, Bao W, Rong Y, Yang H, Bowers K, Yeung E and Kiely M: Genetic variants and the risk of gestational diabetes mellitus: A systematic review. Hum Reprod Update 19: 376-390, 2013.

26. Hildén K, Hanson U, Persson M and Fadl H: Overweight and obesity: A remaining problem in women treated for severe gestational diabetes. Diabet Med 33: 1045-1051, 2016.

27. Macut D, Bjekić-Macut J, Rahelić D and Doknić M: Insulin and the polycystic ovary syndrome. Diabetes Res Clin Pract 130: 163-170, 2017.

28. Boomsma CM, Eijkemans MJ, Hughes EG, Visser GH, Fauser BC and Macklon NS: A meta-analysis of pregnancy outcomes in women with polycystic ovary syndrome. Hum Reprod Update 12: 673-683, 2006.

29. Palomba S, Falbo A, Russo T, Rivoli L, Orio M, Cosco AG, Vero R, Capula C, Tolino A, Zullo F, et al: The risk of a persistent glucose metabolism impairment after gestational diabetes mellitus is increased in patients with polycystic ovary syndrome. Diabetes Care 35: 861-867, 2012.

30. Li G, Huang W, Zhang L, Tian Z, Zheng W, Wang T, Zhang T and Zhang W: A prospective cohort study of early-pregnancy risk factors for gestational diabetes in women with polycystic ovarian syndrome. Diabetes Metab Res Rev 34: e3003, 2018.

31. de Wilde MA, Veltman-Verhulst SM, Goverde AJ, Lambalk CB, Laven JS, Franx A, Koster MP, Eijkemans MJ and Fauser BC: Preconception predictors of gestational diabetes: A multicentre prospective cohort study on the predominant complication of pregnancy in polycystic ovary syndrome. Hum Reprod 29: 1327-1336, 2014.

32. Costrini NV and Kalkhoff RK: Relative effects of pregnancy, estradiol and progesterone on plasma insulin and pancreatic islet insulin secretion. J Clin Invest 50: 992-999, 1971.

33. Lain KY and Catalano PM: Metabolic changes in pregnancy. Clin Obstet Gynecol 50: 938-948, 2007. 
34. Bartha JL, Romero-Carmona R, Torrejon-Cardoso $\mathrm{R}$ and Comino-Delgado R: Insulin, insulin-like growth factor-1 and insulin resistance in women with pregnancy-induced hypertension. Am J Obstet Gynecol 187: 735-740, 2002.

35. Ma RM and Lao TT: Maternal mean arterial pressure and oral glucose tolerance test results. Relationship in normotensive women. J Reprod Med 46: 747-751, 2001.

36. Scholtens DM, Muehlbauer MJ, Daya NR, Stevens RD, Dyer AR, Lowe LP, Metzger BE, Newgard CB, Bain JR, Lowe WL Jr, et al: Metabolomics reveals broad-scale metabolic perturbations in hyperglycemic mothers during pregnancy. Diabetes Care 37: 158-166, 2014.

37. Segura MT, Demmelmair H, Krauss-Etschmann S, Nathan P, Dehmel S, Padilla MC, Rueda R, Koletzko B and Campoy C: Maternal BMI and gestational diabetes alter placental lipid transporters and fatty acid composition. Placenta 57: 144-151,2017.

38. Herrera E and Ortega-Senovilla H: Disturbances in lipid metabolism in diabetic pregnancy-Are these the cause of the problem? Best Pract Res Clin Endocrinol Metab 24: 515-525, 2010.

39. Xia MF, Chen Y, Lin HD, Ma H, Li XM, Aleteng Q, Li Q, Wang D, Hu Y, Pan BS, et al: A indicator of visceral adipose dysfunction to evaluate metabolic health in adult Chinese. Sci Rep 6: 38214, 2016.
40. Butte NF: Carbohydrate and lipid metabolism in pregnancy: Normal compared with gestational diabetes mellitus. Am J Clin Nutr 71 (5 Suppl): 1256S-1261S, 2000.

41. Catalano PM, McIntyre HD, Cruickshank JK, McCance DR, Dyer AR, Metzger BE, Lowe LP, Trimble ER, Coustan DR, Hadden DR, et al: The hyperglycemia and adverse pregnancy outcome study: Associations of GDM and obesity with pregnancy outcomes. Diabetes Care 35: 780-786, 2012.

42. Qiu C, Sorensen TK, Luthy DA and Williams MA: A prospective study of maternal serum C-reactive protein (CRP) concentrations and risk of gestational diabetes mellitus. Paediatr Perinat Epidemiol 18: 377-384, 2004.

43. Lewandowski KC, Stojanovic N, Press M, Tuck SM, Szosland K, Bienkiewicz M, Vatish M, Lewinski A, Prelevic GM and Randeva HS: Elevated serum levels of visfatin in gestational diabetes: A comparative study across various degrees of glucose tolerance. Diabetologia 50: 1033-1037, 2007.

This work is licensed under a Creative Commons Attribution-NonCommercial-NoDerivatives 4.0 International (CC BY-NC-ND 4.0) License. 